\title{
CLINICAL ELECTROCARDIOGRAPHY: THREE-DIMENSIONAL VISUAL AIDS TO TEACHING
}

\author{
BY \\ THOMAS J. RANKIN AND E. GREY DIMOND \\ From the Department of Internal Medicine, School of Medicine, University of Kansas, and the Veterans Administration \\ Hospital, Wichita, Kansas \\ Received April 13, 1951
}

Empiric interpretation of the clinical electrocardiogram is no longer adequate. The fundamental contributions of Wilson and Goldberger have led to the common clinical use of 12 leadsthree standard, three augmented extremity, and six præcordial. If the interpreter is to gain the most knowledge from the information available in these leads, an understanding of the electrical position of the heart in each instance is essential.

Visual aids to the teaching of any spatial principle are of obvious value. Goldberger's book (1949) applies two-dimensional illustrations to the relation of cardiac position and fixed unipolar electrocardiographic leads within the electrical field. This article describes two three-dimensional diagrammatic models of the ventricular heart related to the common fixed leads. One is more applicable to the seminar or individual discussion, the other to the classroom.

\section{DESCRIPTION}

One aid* (Fig. 1) consists of a model roughly fashioned to resemble the ventricles sectioned from the total heart just below the valvular level. Size and shape are those of small normal ventricles. The left ventricular portion with septal wall is carved in soft wood from which a small ventricular cavity is hollowed out. The right ventricular portion is of $1 / 8$ inch transparent plastic, moulded to complete the semi-spherical ventricular structure. For further clarity the left ventricle is painted red, and the septal wall facing the cavity of the right ventricle white. This model is hung at the center of a 10-inch circle of 1/4-inch round steel mounted on a wooden base. The model is joined to its suspending bar by a swivel. The swivel permits the ventricular model to rotate through $360^{\circ}$, to swing in any plane through an arc of $180^{\circ}$, and to remain in whatever position fixed by friction joint. Relative field positions of arm leads and left leg lead are marked on the metal circle by welded tabs labelled R, L, and F. A bar of $1 / 4$ by $3 / 8$-inch plastic is formed into a shallow arc and fastened in horizontal plane to the metal circle from a 9 to 3 o'clock position. Relative field positions of chest leads 1, 2, and 5 are stamped on the plastic. A sliding tab rides the plastic arc to mark any labelled or interpolated position under consideration.

By means of this three-dimensional diagram the ventricular model can be fixed in any position in its electrical field relative to the fixed positions of three limb and antero-lateral chest leads. The electrical source of positive and negative components of the QRS complex as "seen" from any single relatively unipolar lead can be readily visualized. The user must, of course, project from his imagination any alteration of ventricular size or contour attributable to hypertrophy or disease.

The second aid (Fig. 2) $\dagger$ is somewhat similarly conceived, but differently executed. Here

* Made for one of us (T.J.R.) by Mr. Gil Bartlett, Wichita, Kansas, at a cost of $\$ 25.00$ for labour and material. $\dagger$ Made by one of us (E.G.D.), cost of plastic $\$ 8.00$. 
the positions of leads are represented on two large bisecting planes of $1 / 4$-inch transparent plastic in vertical and horizontal positions. The vertical plane is triangular and measures 18 inches on each side. The horizontal plane is a circle 18 inches in diameter. The triangle rests in and can be removed from the circle which is supported on a wooden tripod. The center of the bisection of the plastic planes is cut out to accommodate a hollow hemisphere made from half a rubber ball. This represents the ventricles and is painted one half blue and one half red to indicate the right and left ventricle. A separate plastic "card" is shaped to fit loosely within the ball to represent the

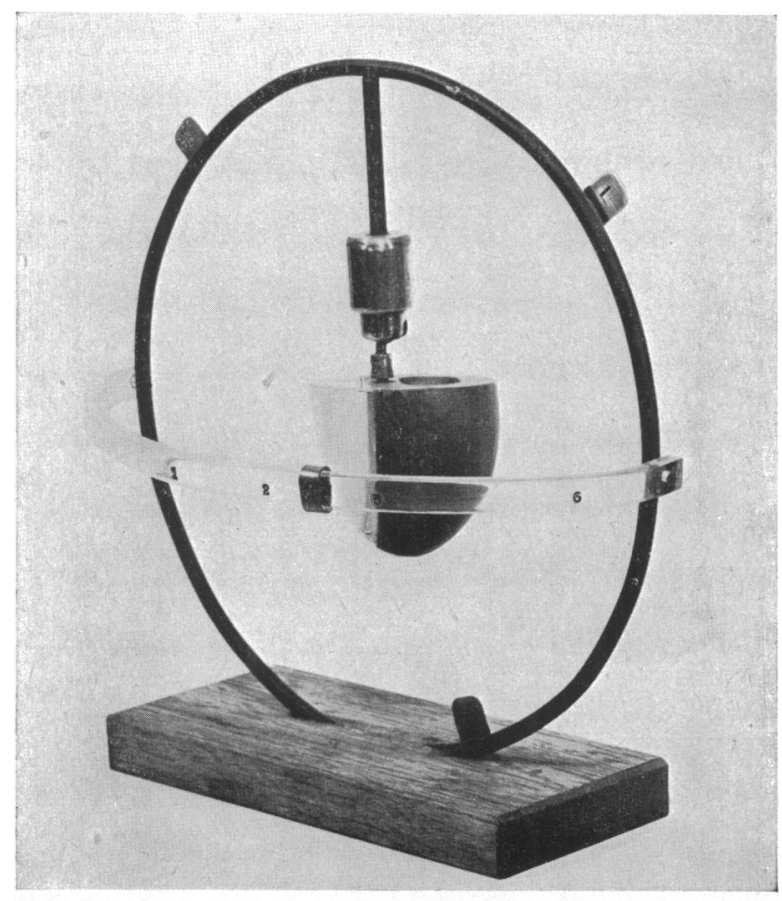

FIG. 1.-Ventricle and ring model. See text and subsequent figures for application.

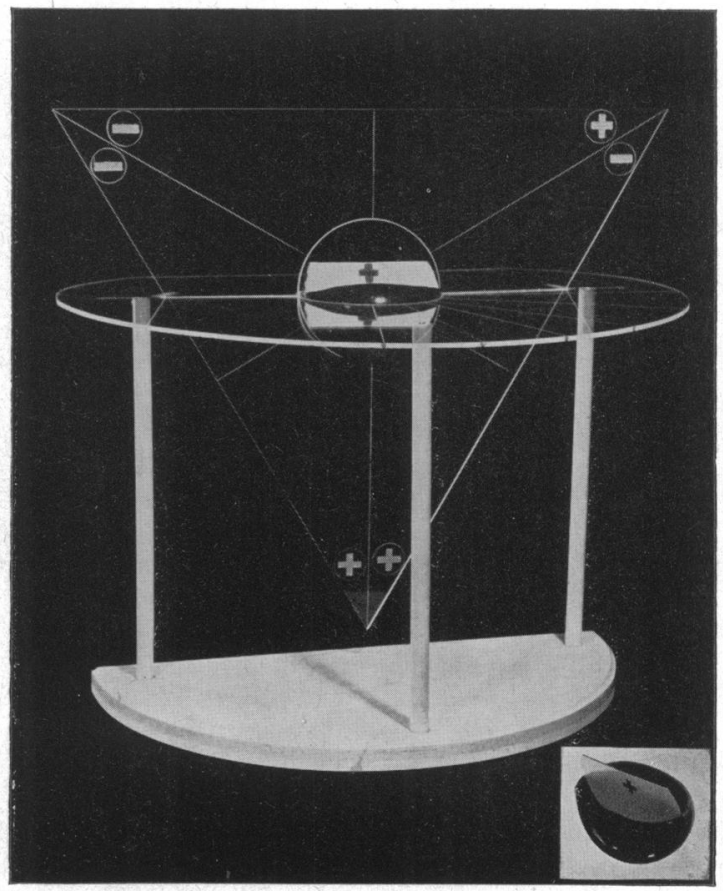

FIG. 2.-Plastic plane model. See text for application.

ventricular septum. This is painted black on one side apd white on the other to indicate negativity and positivity. The "septum" within the ventricles and the "ventricles" within the bisecting planes can be placed in any position dictated by the circumstances of demonstration or the pattern of electrocardiographic tracings. Positions of chest leads 1 to 6 are indicated by radiating lines drawn on the horizontal plane. The corners of the triangle represent the limb leads and are marked with positive and negative symbols. These symbols with lines bisecting the triangle from each corner serve to illustrate the principles of Einthoven's triangle when this plane is lifted from the supporting horizontal one.

\section{USE AND EXAMPLES}

Excepting as they can be shown to be combinations of lead potentials the standard leads I, II, and III are not used in model positioning. Fig. 3 and 4 show the first model with the ventricles in vertical and horizontal positions, with appropriate electrocardiograms.

In the vertical heart the position is achieved in the following manner. Lead aVR shows a QS or Qr pattern typical of a lead facing the ventricular cavity, so also does lead aVL. Therefore 


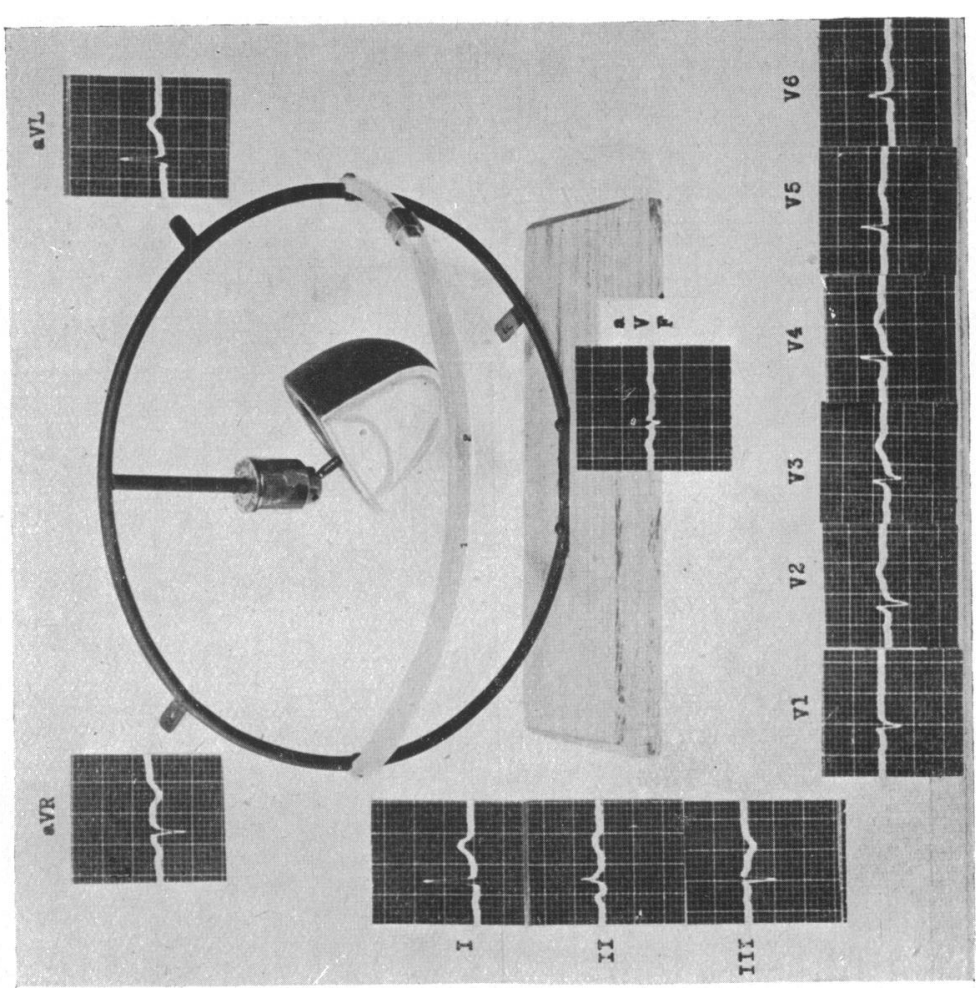

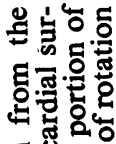

통ํํำ

\% ส

2 1

$\rightarrow$

든영

娄到管

西

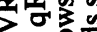

$\approx \pi$ \%

+ 质

还市

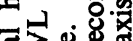

ฐึ兀。

5

대잉

놀

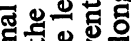

웅년도

$\mathrm{Z} \geq 0$.

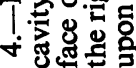

空
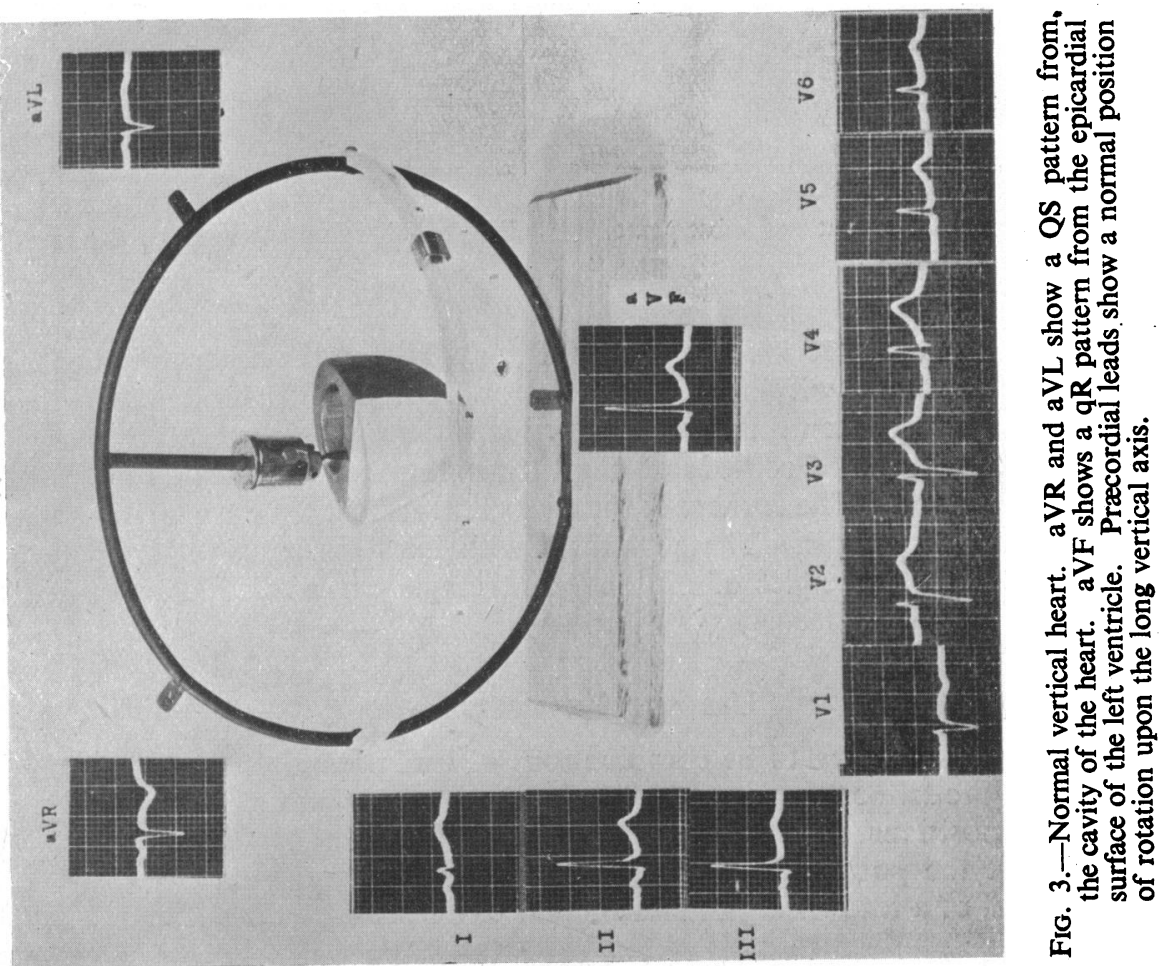

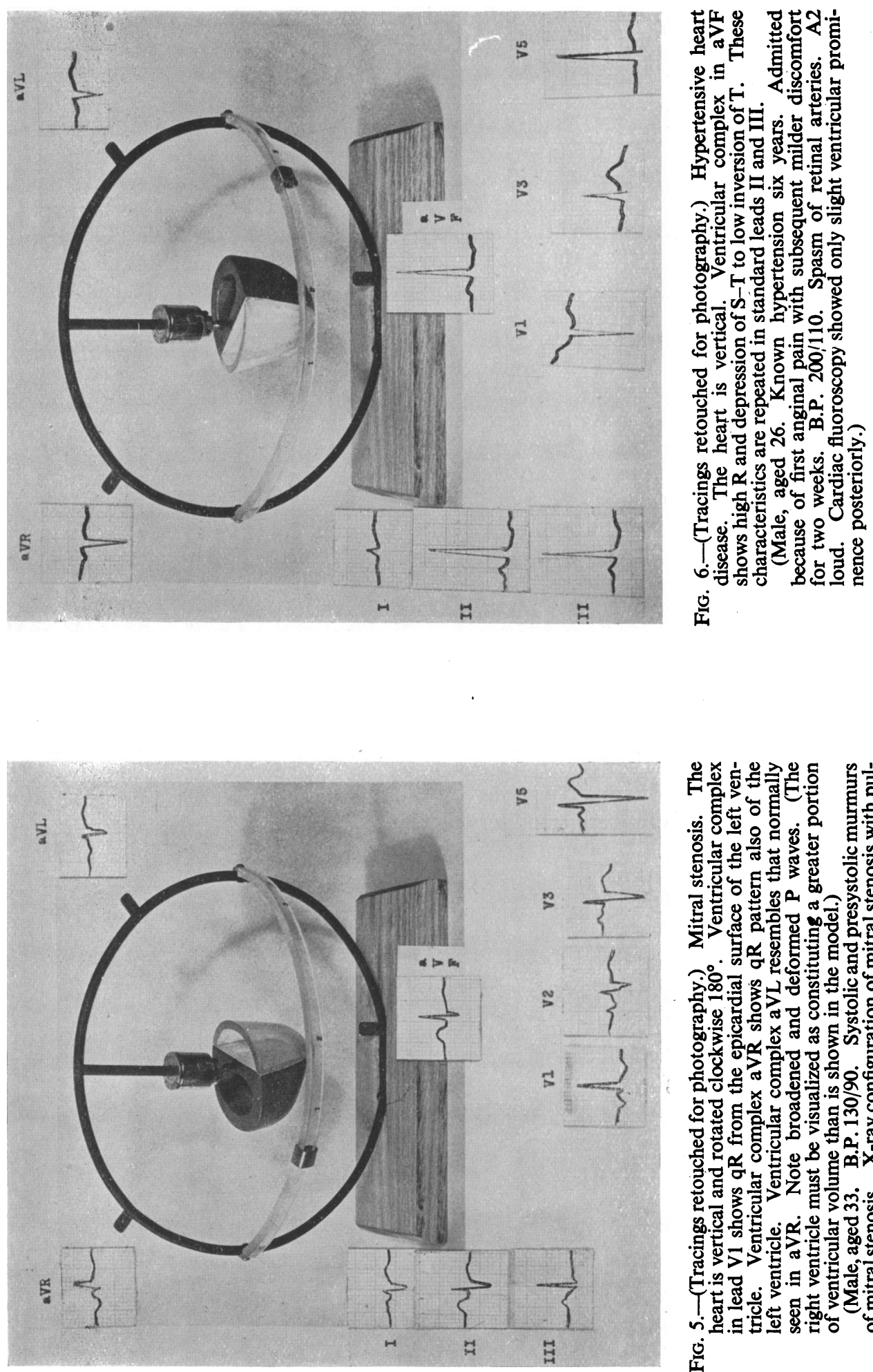

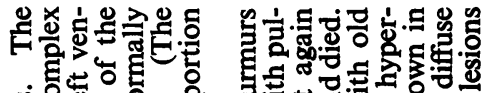

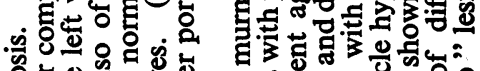

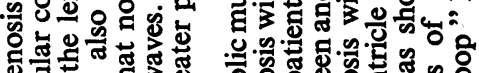

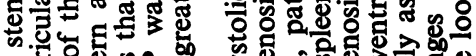

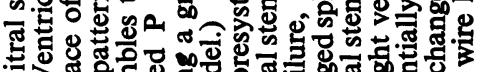

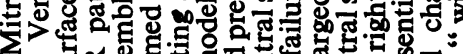

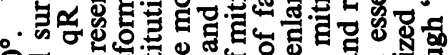

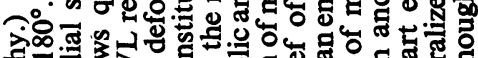

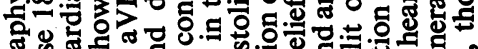

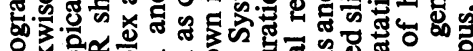
웅

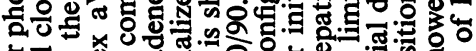
후영영.

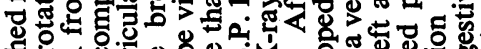

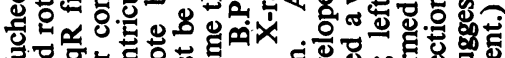

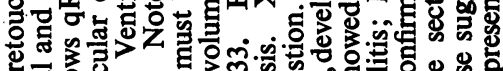

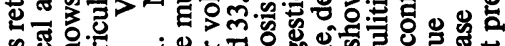

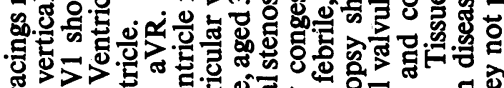
象可.

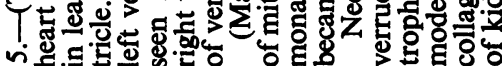
它 
the model is positioned with the ventricular cavity up. This vertical position is confirmed by and further drawn from a $\mathrm{qR}, \mathrm{qRs}, \mathrm{R}$ or Rs pattern in lead aVF. The predominant $\mathrm{R}$ shows $\mathrm{aVF}$ alone among the limb leads to face the epicardial surface of the left ventricle.

In the horizontal heart aVF also shows a QS pattern. The beginning position of the model is also with the cavity of the ventricles up. Lead aVL, however, shows a qR pattern. The small initial $\mathrm{q}$ indicates the lead to be facing the negative or left side of the septum, the prominent $R$ shows it to be facing the epicardial surface of the left ventricle. The model is tilted so that the lateral surface of the left ventricle faces the tab marked $L$. The horizontal position is confirmed and further drawn from an $\mathrm{rSr}$ pattern in lead aVF. The small $\mathrm{r}$ indicates the lead to be facing the positive or right side of the septum, the $S$ that the summation of major potential is of the endocardial surface of the left ventricle " seen " through the right. Therefore, the apical surface of the right ventricle should be positioned to face the tab $F$.

In either instance, horizontal or vertical, the rotation of the heart upon its vertical axis can be determined by reference to the chest leads. In normal positions V1 should face the right ventricle and show an rS pattern, lead V6 should face the epicardial surface of the left ventricle and show a $\mathrm{qR}$ pattern. By consideration of the shift of pattern through V2-V5 the model can be placed in approximate clockwise or counterclockwise rotation upon its vertical axis.

These oversimplified normal positions are seen with reasonable frequency in clinical tracings and serve as reference points for the solution of less obvious cardiac positions.

Fig. 5 shows the cardiac position by model and the clinical tracing in a case of mitral stenosis. Clockwise rotation is so extreme that lead V1 shows a $\mathrm{qR}$ pattern from the epicardial surface of the left ventricle.

One of the most pertinent clinical demonstrations of the model is seen in left heart " strain " in the vertical heart (Fig. 6). Here the abnormal S-TT segment change is seen in aVF where the septal $\mathrm{q}$ and prominent $\mathrm{R}$ indicate the epicardial surface of the left ventricle facing the diaphragm, with a vertical heart. The abnormality of lead aVF is reflected in standard leads II and III. Empiric interpretation of standard leads could lead to the inference of right heart " strain."

Further elaboration by example is beyond the purpose of this presentation. The authors have found the ring and ventricle model very useful in ward teaching by specific clinical application.

The large plastic model has been a useful visual aid particularly for beginning instruction with groups who have been familiar with the three standard leads but have difficulty in visualizing the relationship of the limb leads as a frontal plane section and the chest leads as a horizontal plane section. The triangle is lifted out of the model and held in front of the lecturer. When the group has assimilated this concept, the triangle is returned to the model and three-dimensional relationships are developed.

\section{CONCLUSION}

Two three-dimensional models of the cardiac ventricles in relation to limb and chest electrocardiographic leads are described. Their value to teaching is noted. Examples of their application are presented.

\section{REFERENCE}

Goldberger, E. (1949). Unipolar Lead Electrocardiography, 2nd ed., Lea and Febiger, Philadelphia. 